\title{
Future Trends of Diabetes: Changing Basic Concepts Towards More Rational Attitudes
}

Alkhatib $\mathrm{J}^{1,2^{*}}$

${ }^{1}$ Department of Legal Medicine, Toxicology of Forensic Science and Toxicology, School of Medicine, Jordan University of Science and Technology, Jordan

${ }^{2}$ International Mariinskaya Academy, department of medicine and critical care, department of philosophy, Academician secretary of department of Sociology

Corresponding Author: Ahed J Alkhatib

Address: Department of Legal Medicine, Toxicology of Forensic Science and Toxicology, School of Medicine, Jordan University of Science and Technology, Jordan; E-mail: ahed.alkhatib64@yahoo.com, ajalkhatib@just.edu.jo, drahedalkhatib@yahoo.com

Received date: 21 October 2019; Accepted date: 25 October 2019; Published date: 30 October 2019

Citation: Alkhatib J. Future Trends of Diabetes: Changing Basic Concepts Towards More Rational Attitudes. Diab Res Open Access. 2019 Oct 30;1(1):5-6.

Copyright (C) 2019 Alkhatib J. This is an open-access article distributed under the Creative Commons Attribution License, which permits unrestricted use, distribution, and reproduction in any medium, provided the original work is properly cited.

\section{Keywords}

Diabetes; Diabetic Patients; Type-1 Diabetes; Type-2 Diabetes

In this editorial, I would like to go through some philosophical aspects in conceptual formulations. Diabetes has been defined as part of metabolic syndrome. Diabetes is associated with insulin deficiency either in concentration or function. This is the classical and traditional picture associated with diabetes type 2. Diabetic complications are also associated with these concepts involving the metabolic points of view.

Over more than 15 years of research in diabetes, I have found other parameters are likely to impact our concepts regarding diabetes that may shape future management of diabetes. The first change in concepts was if diabetes is initiated in other organs or tissues far away from pancreas. Our studies showed the involvement of alterations in molecular changes in white matter. We found that white matter functional alterations play a significant role in initiating episodes of diabetes type 1 animal models (rats). We found that inducible nitric oxide synthase is significantly expressed in white matter compared with control group. At the same time, we found significant less expression of $\mathrm{HSP}_{70}$ in white matter of diabetic rat compared with control group. We reached a conclusion that neuro-inflammatory conditions may play a new role in the development of diabetes. This also may explain the development of diabetic neuropathies. We conducted further studies that showed the same pattern of changes in other organs including liver, kidney, and skin.

Because these changes are constant and follow kinetic patterns, we conducted other battery of experiments that showed exercise can change the molecular alteration in white matter which can improve the functional status of white matter and other organs involved in our previous studies including liver, kidney, and skin. Accordingly, we reached a conclusion that diabetes is neurological disorder more than a metabolic disorder. 

2019 Oct 30;1(1):5-6.

\section{Editorial}

This hypothesis is further supported by using big data. We found that as the glucose level increases, the insulin level increases. This is a proportional relationship. We analyzed data of 3000 diabetic patients from Germany and India. We subdivided data as the following patterns: normal, pre-diabetic, and diabetic groups according to glucose level. We found that insulin level was significantly increased as the pattern has changed from normal, pre-diabetic, to diabetic patterns. These results raised the question of rationality of using insulin in treating patients with type 2 diabetes.
Increased insulin levels have health adverse effects on the body including heart disease, obesity and cancer. Accordingly, the irrational use or initiation of insulin treatment is a process associated with health risks.

From this editorial point of view, we think that measurement of insulin levels should go side by side with measuring both glucose levels and $\mathrm{HbA1C}$ levels for appropriate control of diabetic status. We suggest that medical manufacturing companies to produce devices that can measure the three tests glucose, $\mathrm{HbA}_{1} \mathrm{C}$, and insulin at the same time. 